\title{
VII. Ventilación no invasiva en pacientes con daño pulmonar agudo
}

\author{
RODRIGO SOTO F.*, CÉSAR MAQUILÓN O.** y FERNANDO SALDÍAS P.***
}

\section{Noninvasive ventilation in patients with acute lung injury and hypoxemic respiratory failure}

La Ventilación Mecánica No Invasiva (VNI) en los pacientes con insuficiencia respiratoria aguda se ha asociado a mejoría en el intercambio gaseoso y alivio de la disnea por mecanismos fisiológicos previamente descritos. Sin embargo, en los pacientes con daño pulmonar agudo (ALI) y síndrome de dificultad respiratoria aguda (SDRA) existe escasa información sobre los efectos potencialmente benéficos de la VNI comparado con la ventilación mecánica convencional. El SDRA es la forma más grave de falla respiratoria aguda manejada en las unidades de cuidado crítico, con morbi-mortalidad elevada, caracterizada por aumento de la permeabilidad y ruptura de la barrera alvéolo-capilar pulmonar e inflamación de los espacios aéreos, asociado a cambios sistémicos que afectan significativamente la evolución de los enfermos y dificultan la interpretación de los resultados de los estudios que evalúan la eficacia de las distintas técnicas ventilatorias.

Pregunta: ¿La VNI reduce la mortalidad y la necesidad de VM invasiva en pacientes con daño pulmonar agudo o SDRA?

Pacientes: Pacientes con daño pulmonar agudo o SDRA asociado a falla respiratoria hipoxémica que necesitan asistencia ventilatoria.

Intervención: VNI aplicada a pacientes con falla respiratoria hipoxémica y daño pulmonar agudo o SDRA.

\section{Resumen de la evidencia}

Garpestad y cols, advierten que la categoría diagnóstica "falla respiratoria aguda" está constituida por un grupo heterogéneo de enfermedades cardiorrespiratorias, y que el beneficio im- portante obtenido con la VNI en pacientes con enfermedad pulmonar obstructiva crónica avanzada, edema pulmonar cardiogénico y pacientes inmunosuprimidos con infecciones respiratorias, no necesariamente puede ser reproducido en otras entidades clínicas ${ }^{1}$. Por razones obvias, no es posible realizar estudios "doble ciego" que evalúen la eficacia de la VNI en distintos contextos clínicos, y la necesidad de cooperación para la implementación del procedimiento introduce sesgos en el análisis y la interpretación de los resultados.

Antonelli y cols, en un estudio clínico controlado examinaron la eficacia de la VNI en 64 pacientes con falla respiratoria aguda severa comparado con la ventilación mecánica convencional. Este estudio excluyó a los pacientes con EPOC y las causas de daño pulmonar agudo fueron similares en ambos grupos: neumonía, trauma, edema pulmonar cardiogénico, otras. La sobrevida en la UCI fue $53 \%$ en el grupo manejado con terapia convencional (17/32) y $72 \%$ en el grupo tratado con VNI (23/32). Por otra parte, en la mayoría de los casos la VNI se asoció a mejoría significativa de la $\mathrm{PaO}_{2} / \mathrm{FiO}_{2}$ al cabo de una hora de tratamiento y sólo un tercio (10/ 32) requirieron intubación, mientras que todos estaban intubados en el grupo control. Veinte y dos pacientes tratados con VNI sobrevivieron en el hospital (68\%) comparado con 16 pacientes $(50 \%)$ en el grupo control (falleció un paciente en cada grupo fuera de la UCI).

Los pacientes manejados con VNI tuvieron menos complicaciones (sinusitis, neumonías, lesiones traumáticas de la vía aérea), la estadía promedio en ventilación mecánica disminuyó de

Jefe Servicio de Enfermedades Respiratorias, Hospital de la Fuerza Aérea de Chile.

** Residente de la Unidad de Cuidados Intermedios del Instituto Nacional del Tórax y Unidad de Cuidados Intensivos de la Clínica Dávila.

*** Profesor Asociado de Medicina, Departamento de Enfermedades Respiratorias, Pontificia Universidad Católica de Chile. 
6 a 3 días y la permanencia en la UCI fue más breve (VNI: $9 \pm 7$ días vs VM: $16 \pm 17$ días). Los ventiladores empleados en el estudio fueron PB 7200 y Servo 900 C.

Luego, Antonelli y cols, en un estudio de cohorte multicéntrico en una población de 479 pacientes con SDRA, evaluaron la eficacia de la VNI en 147 pacientes que no estaban intubados en la admisión a la UCI, en ellos se logró evitar la intubación en el 54\% de los pacientes, disminuyendo la morbilidad, estadía en ventilador y mortalidad en la $\mathrm{UCI}^{3}$. Es importante mencionar que este resultado fue obtenido en pacientes con SDRA menos graves (no intubados en la admisión), y así se logró una mortalidad en UCI de $6 \%$ comparado con $53 \%$ en el grupo ventilado. La cifra de $6 \%$ está fuera de cualquier reporte conocido de mortalidad en SDRA, y sólo se entiende bajo la consideración de que se trata de un grupo muy especial, no representativo del universo de pacientes con SDRA. En esta experiencia, los autores examinaron los factores de riesgo de fracaso de la VNI, destacando la edad avanzada, la necesidad de PEEP y presión de soporte elevados, un índice de gravedad SAPS mayor de 35 y una relación $\mathrm{PaO}_{2} / \mathrm{FIO}_{2}$ menor de $175 \mathrm{mmHg}$ al cabo de una hora de tratamiento.

En un estudio clínico prospectivo de una cohorte de 524 pacientes con falla respiratoria aguda que requerían ventilación mecánica, Demoule y cols, demostraron que la aplicación de VNI tuvo un impacto favorable en los tres grupos de pacientes, EPOC exacerbado, edema pulmonar cardiogénico y la categoría que denominaron falla respiratoria "de novo" (299 pacientes) $)^{4}$. Sin embargo, en este último grupo el índice de fracaso de la VNI fue mayor y el intento frustro de manejo con VNI se asoció a un aumento significativo de la mortalidad (OR 3,24 , IC95\% 1,61-6,25). De modo que los autores ponen una nota de cautela ante la indicación de VNI en este contexto clínico.

Honrubia y cols, examinaron la eficacia de la VNI en 64 pacientes con falla respiratoria aguda comparado con la VM convencional, se incluyeron en este estudio 38 pacientes con EPOC exacerbado ${ }^{5}$ Los autores demostraron una reducción del riesgo relativo de intubación, pero no observaron cambios significativos en la mortalidad, tasa de complicaciones, estadía en UCI, ni complejidad de los cuidados medido por TISS.

En un metanálisis efectuado por Keenan y cols, que examinaron ocho estudios de VNI en pacientes con SDRA, que no requirieron intubación inmediata, observaron reducción significativa de la tasa de intubación, estadía en la UCI y letalidad en el hospital ${ }^{6}$. Destacando la gran heterogeneidad de las poblaciones estudiadas, el estudio incluyó pacientes inmunosuprimidos y postoperados, y recomienda cautela en la interpretación de los resultados de la VNI en pacientes con falla respiratoria hipoxémica. Además, se menciona la eventual ventaja de la VNI en pacientes postoperados e inmunosuprimidos.

En un estudio que examinó la eficacia de la ventilación mecánica en decúbito prono precoz y prolongada en pacientes con SDRA, Mancebo y cols, destacaron que la mortalidad en los pacientes que recibieron VNI antes de la intubación y conexión a ventilador fue más elevada $(60,7 \%$ vs $42,5 \%$ en aquellos que no la recibieron, $\mathrm{p}=$ $0,055)^{7}$.

En una revisión reciente del tema, Phillips y Hyzy, afirmaron que la VNI puede evitar la intubación en algunos pacientes, pero aún existen datos insuficientes para recomendar este procedimiento en el manejo rutinario de los pacientes con $\mathrm{SDRA}^{8}$. En definitiva la mayoría de los enfermos requieren intubación y es necesario no dilatar o diferir la inminente intubación, lo cual ensombrece el pronóstico vital. En la misma línea de pensamiento, Garpestad editorializa el estudio de Honrubia, destacando que la VNI no debe ser considerada un recurso estándar para el manejo de la falla respiratoria aguda y puede ensayarse siempre que se asegure la intubación necesaria dentro de las primeras 2-3 horas de manejo, si éste resulta frustro ${ }^{9}$.

Hill y cols, examinaron los principales factores predictores de fracaso de la VNI: SDRA, neumonía grave, shock, acidosis metabólica, SAPS > 35 y $\mathrm{PaO}_{2} / \mathrm{FIO}_{2}$ inicial menor de 175 $\mathrm{mmHg}$ luego de una hora de tratamiento ${ }^{10}$.

Peter y cols, examinaron la eficacia de la VNI en pacientes con falla respiratoria aguda en un metanálisis de 15 estudios, describen una reducción de la mortalidad de $8 \%$, de la necesidad de intubación de $19 \%$, y un acortamiento de la estadía en UCI de 2,74 días en el grupo de pacientes sometidos a VNI comparado con la terapia estándar ${ }^{11}$. Sin embargo, el grupo que más se benefició fueron los pacientes con enfermedad pulmonar obstructiva crónica exacerbados, mientras que los pacientes con falla respiratoria de "causa mixta" sólo mostraron reducción de la necesidad de intubación y conexión a ventilador.

La guía clínica IDSA-ATS para el manejo de la neumonía comunitaria del adulto recomienda evaluar la aplicación de VNI en los pacientes con hipoxemia y SDRA, salvo que los pacientes requieran intubación inmediata, la relación $\mathrm{PaO}_{2} /$ 
Tabla 1. Eficacia clínica de la ventilación no invasiva en pacientes con daño pulmonar agudo o síndrome de dificultad respiratoria aguda

\begin{tabular}{|c|c|c|c|}
\hline Variables & Peter y cols. ${ }^{11}$ & Antonelli y cols. ${ }^{2}$ & Antonelli y cols. ${ }^{3}$ \\
\hline $\begin{array}{l}\text { Tipo de } \\
\text { estudio }\end{array}$ & Meta-análisis & $\begin{array}{l}\text { Prospectivo, aleatorio, } \\
\text { controlado }\end{array}$ & $\begin{array}{l}\text { Cohorte, prospectivo, } \\
\text { multicéntrico }\end{array}$ \\
\hline Período & & 1995-1996 & 2002-2004 \\
\hline Lugar & UCI & UCI & UCI \\
\hline Modalidad & & CPAP+PS & $\mathrm{CPAP}+\mathrm{PS}$ \\
\hline Interface & Nasal o facial & Oronasal & Oronasal y casco \\
\hline Pacientes & $\begin{array}{l}15 \text { estudios controlados de } \\
\text { VNI vs control }\end{array}$ & $\begin{array}{l}\text { N: } 32 \text { IRA en VNI } \\
\text { CT: } 32 \text { IRA en VM }\end{array}$ & $\begin{array}{l}\text { N: } 147 \text { SDRA con VNI } \\
\text { CT: } 332 \text { SDRA con VM }\end{array}$ \\
\hline $\begin{array}{l}\text { Fracaso de } \\
\text { la VNI }\end{array}$ & & $\begin{array}{l}\text { 10/32 pacientes en el grupo de } \\
\text { VNI fueron intubados }(31 \%)\end{array}$ & $\begin{array}{l}68 \text { pacientes manejados con } \\
\text { VNI fueron intubados }(46 \%)\end{array}$ \\
\hline $\begin{array}{l}\text { Efecto de } \\
\text { la VNI }\end{array}$ & $\begin{array}{l}\text { Reducción de la mortalidad en } \\
\text { UCI de } 17 \% \text { (IC95\%: 8-26\%) } \\
\text { Reducción del riesgo de intuba- } \\
\text { ción: } 23 \% \text { (IC95\%: 10-35\%) } \\
\text { Reducción de la estancia en } \\
\text { UCI de 1,9 días (IC95\%: } \\
\text { 1-2,9 días) } \\
\text { Reducción de la mortalidad en } \\
\text { el hospital de } 10 \% \text { (IC 95\%: } \\
0,73-1,07 \text { ) }\end{array}$ & $\begin{array}{l}\text { Disminución de la mortalidad } \\
\text { en la UCI }(\mathrm{OR}, 0,4 ; \text { IC } 95 \% \text { : } \\
0,1-1,4 ; p=0,19) \\
\text { Períodos más cortos de ventila- } \\
\text { ción }(\mathrm{p}=0,006) \text { y permanencia } \\
\text { en la UCI }(\mathrm{p}=0,002) \\
\text { Menos complicaciones asocia- } \\
\text { das a la ventilación }(38 \% \text { vs } \\
66 \%, p=0,02)\end{array}$ & $\begin{array}{l}\text { Se evitó la intubación en } 54 \% \\
\text { de los pacientes } \\
\text { La mortalidad en la UCI dismi- } \\
\text { nuyó en los pacientes sometidos } \\
\text { a VNI que no se intubaron } \\
\text { ( } 6 \% \text { vs } 36 \% \text { en aquellos que se } \\
\text { intuban) } \\
\text { La mortalidad en el hospital dis- } \\
\text { minuyó en el grupo con VNI: } \\
15 \% \text { vs VM: } 38 \%\end{array}$ \\
\hline
\end{tabular}

Nota: UCI: unidad de cuidados intensivos, CPAP-PS: presión positiva continua en la vía aérea-presión de soporte, IRA: insuficiencia respiratoria aguda, SDRA: síndrome de dificultad respiratoria aguda, VNI: ventilación no invasiva, VM: ventilación mecánica.

$\mathrm{FIO}_{2}$ sea menor de $150 \mathrm{mmHg}$ o tengan infiltrados pulmonares bilaterales en la radiografía de tórax $^{12}$.

\section{Conclusión}

La información disponible sobre la eficacia de la VNI en pacientes con SDRA/ALI es escasa y confusa, esta confusión deriva de la heterogeneidad en las características clínicas de los grupos con falla respiratoria aguda examinados (Tabla 1). La inclusión de pacientes con edema pulmonar cardiogénico, EPOC e inmunodepresión favorecen los resultados de la terapia, ya que en estas patologías es donde más éxito ha tenido esta intervención. Además, en las distintas series se seleccionan los casos de menor gravedad inicial y en ese contexto clínico se extraen conclusiones.

La VNI puede reducir el riesgo de intubación, complicaciones y estadía en la UCI cuando es exitosa, pero en pacientes con SDRA la posibilidad de fracaso del procedimiento oscila entre 50 y $70 \%$, y el fracaso se ha asociado a un incremento de la mortalidad. Parece recomendable tener presentes los criterios clínicos predictores de fracaso, evitando diferir la intubación necesaria más allá de 1 a 3 horas de observación. Pudiera haber algún beneficio en pacientes con falla respiratoria parcial y compromiso sistémico de bajo grado.

\section{Efecto potencial}

En algunos pacientes con falla respiratoria hipoxémica y SDRA, el uso de VNI podría reducir la necesidad de intubación endotraqueal y las complicaciones asociadas a la ventilación mecánica.

\section{Evaluación del costo/beneficio}

De acuerdo a la información disponible, no es posible asegurar que la VNI en pacientes con falla respiratoria hipoxémica y SDRA sea costo/ efectiva. Se necesita de estudios clínicos prospectivos, controlados, multicéntricos, que permitan precisar las indicaciones, rendimiento, limitaciones y poblaciones de pacientes que se beneficiarán de esta modalidad terapéutica.

\section{Grado de recomendación}

Se recomienda intentar aplicar VNI en pacientes con ALI-SDRA inicial cuando existe falla de un solo sistema e hipoxemia moderada (recomendación débil, evidencia de baja calidad). 
Se recomienda no retardar la intubación de entrada en pacientes con SDRA establecido, compromiso multisistémico y en pacientes con alto riesgo de fracaso (recomendación fuerte, evidencia de mediana calidad).

\section{Bibliografía}

1.- GARPESTAD E, BRENNAN J, HILL N S. Noninvasive ventilation for critical care. Chest 2007; 132: 71120.

2.- ANTONELLI M, CONTI G, ROCCO M, BUFI M, DE BLASI RA, VIVINO $\mathrm{G}$, et al. A comparison of noninvasive positive-pressure ventilation and conventional mechanical ventilation in patients with acute respiratory failure. N Engl J Med 1998; 339: 429-35.

3.- ANTONELLI M, CONTI G, ESQUINAS A, MONTINI L, MAGGIORE S M, BELLO G, et al. A multiplecenter survey on the use in clinical practice of noninvasive ventilation as a first-line intervention for acute respiratory distress syndrome. Crit Care Med 2007; 35: 18-25.

4.- DEMOULE A, GIROU E, RICHARD J C, TAILLE S, BROCHARD L. Benefits and risks of success or failure of noninvasive ventilation. Intensive Care Med 2006; 32: $1756-65$.

5.- HONRUBIA T, GARCÍA LÓPEZ F J, FRANCO N, MAS M, GUEVARA M, DAGUERRE $M$, et al.
Noninvasive vs conventional mechanical ventilation in acute respiratory failure: a multicenter, randomized controlled trial. Chest 2005; 128: 3916-24.

6.- KEENAN S P, SINUFF T, COOK D J, HILL N S. Does noninvasive positive pressure ventilation improve outcome in acute hypoxemic respiratory failure? A systematic review. Crit Care Med 2004; 32: 251623.

7.- MANCEBO J, FERNÁNDEZ R, BLANCH L, RIALP G, GORDO F, FERRER M, et al. A multicenter trial of prolonged prone ventilation in severe acute respiratory distress syndrome. Am J Respir Crit Care Med 2006; 173: 1233-9.

8.- PHILIPS K, HYZY R. Noninvasive ventilation. Uptodate, Jan 2007.

9.- GARPESTAD E, HILL N. Noninvasive ventilation for acute respiratory failure: but how severe? Chest 2005; 128: 3790-1.

10.- HILL N S, BRENNAN J, GARPESTAD E, NAVA S. Noninvasive ventilation in acute respiratory failure. Crit Care Med 2007; 35: 2402-7.

11.- PETER J V, MORAN J L, PHILLIPS-HUGHES J, WARN D. Noninvasive ventilation in acute respiratory failure-a meta-analysis update. Crit Care Med 2002; 30: 555-62.

12.- MANDELL L A, WUNDERINK R G, ANZUETO A, BARTLETT J G, CAMPBELL G D, DEAN N C, et al; Infectious Diseases Society of America; American Thoracic Society. Infectious Diseases Society of America/American Thoracic Society consensus guidelines on the management of community-acquired pneumonia in adults. Clin Infect Dis 2007; 44 (Suppl 2): S27-72.

Correspondencia a:

Dr. Rodrigo Soto F.

E-mail: rsoto955@hotmail.com 